\title{
A compact and mobile hybrid C-arm scanner for simultaneous nuclear and fluoroscopic image guidance
}

\author{
Martijn M. A. Dietze ${ }^{1,2}$ (1) Britt Kunnen ${ }^{1,2} \cdot$ Frank Brontsema $^{1} \cdot$ Pascal Ramaekers $^{1} \cdot$ Casper Beijst $^{1} \cdot$ Maryam $^{\text {Afifah }}{ }^{1} \cdot$ \\ Arthur J. A. T. Braat ${ }^{1} \cdot$ Marnix G. E. H. Lam ${ }^{1} \cdot$ Hugo W. A. M. de Jong ${ }^{1}$
}

Received: 12 February 2021 / Revised: 22 March 2021 / Accepted: 27 April 2021 / Published online: 16 June 2021

(C) The Author(s) 2021

\begin{abstract} phantom with a mixture of iodinated contrast and ${ }^{99 \mathrm{~m}} \mathrm{Tc}$. qualities. Nevertheless, IXSI realizes unique image guidance that may be beneficial for several types of procedures.

Key Points

- IXSI can perform time-resolved planar (2D) simultaneous fluoroscopic and nuclear imaging.

- IXSI can perform SPECT/CBCT imaging (3D) inside the intervention room.
\end{abstract}

Purpose This study evaluates the performance of a mobile and compact hybrid C-arm scanner (referred to as IXSI) that is capable of simultaneous acquisition of 2D fluoroscopic and nuclear projections and 3D image reconstruction in the intervention room. Results The impact of slightly misaligning the IXSI modalities (in an off-focus geometry) was investigated for the reduction of the fluoroscopic and nuclear interference. The 2D and 3D nuclear image quality of IXSI was compared with a clinical SPECT/CT scanner by determining the spatial resolution and sensitivity of point sources and by performing a quantitative analysis of the reconstructed NEMA image quality phantom. The 2D and 3D fluoroscopic image of IXSI was compared with a clinical CBCT scanner by visualizing the Fluorad A+D image quality phantom and by visualizing a reconstructed liver nodule phantom. Finally, the feasibility of dynamic simultaneous nuclear and fluoroscopic imaging was demonstrated by injecting an anthropomorphic

Conclusion Due to the divergent innovative hybrid design of IXSI, concessions were made to the nuclear and fluoroscopic image

Keywords Hybrid imaging $\cdot$ Scintigraphy $\cdot$ Fluoroscopy $\cdot$ SPECT $\cdot$ Cone-beam CT

\section{Abbreviations}

CBCT Cone-beam computed tomography

CT Computed tomography

FOV Field of view

FWHM Full width at half maximum

IXSI Interventional $\mathrm{x}$-ray and scintigraphy imaging

SPECT Single-photon emission computed tomography

Martijn M. A. Dietze

m.m.a.dietze@umcutrecht.nl

1 Radiology and Nuclear Medicine, University Medical Center Utrecht and Utrecht University, P.O. Box 85500, 3508

GA Utrecht, The Netherlands

2 Image Sciences Institute, University Medical Center Utrecht and Utrecht University, P.O. Box 85500, 3508

GA Utrecht, The Netherlands

\section{Introduction}

To date, imaging in the intervention room has primarily consisted of using fluoroscopy for mapping of the anatomy or using nuclear imaging for localization of tumors and potential metastasis. Following the success of diagnostic hybrid imaging, we believe that there may be added value in combining these two interventional modalities: i.e., performing fluoroscopy simultaneously with nuclear imaging. Such a hybrid modality would impact two types of procedures.

Firstly, the procedures that are currently done under nuclear imaging guidance but may benefit from additional fluoroscopic imaging. These procedures include sentinel node procedures [1] and parathyroidectomies [2]. Simultaneous acquisition of anatomical information in these procedures would improve the interpretability of the nuclear images. This may realize a similar benefit as is achieved in diagnostic hybrid imaging (e.g., SPECT/CT, PET/CT). 
Secondly, the procedures that are currently done under fluoroscopic image guidance but may benefit from additional nuclear imaging. For instance, radioactive particles are injected through a catheter in intra-arterial peptide receptor radionuclide therapy [3] and radioembolization [4, 5]. Simultaneous acquisition of nuclear imaging in these procedures will provide direct feedback on the acquired activity distribution, which improves the safety and the efficacy. Additional nuclear imaging may furthermore aid myocardial interventions [6] by providing complementary information for the localization of a defect.

We have previously proposed an innovative detector assembly that realizes hybrid nuclear and fluoroscopic imaging in the intervention room and evaluated this design through prototype systems [7-9]. The aim of the current study is to evaluate the imaging performance of the first scanner configuration that has been approved by the local ethics committee to be evaluated in existing clinical workflows.

\section{Materials and methods}

\section{IXSI}

The developed scanner will be referred to as IXSI (Interventional X-ray and Scintigraphy Imaging). The detector stack of IXSI (see Fig. 1a) consists of an X-ray flat panel detector (Pixium 3040; Trixell) that is positioned in front of a gamma camera (P3000; Inter Medical) that is mounted with a cone-beam collimator (Nuclear Fields). The detector stack is placed on a custom-made C-arm (Indes) together with an $\mathrm{x}$ ray tube (Veradius; Philips Healthcare) on the opposite side. The x-ray tube is placed in the focal point of the cone-beam collimator $(105 \mathrm{~cm})$, which results in simultaneously overlapping nuclear and fluoroscopic projections (see Fig. 1b). The $\mathrm{C}$-arm can translate in two dimensions while rotating and can perform parameterized non-circular orbits. A collision detector is present on top of the detector stack to ensure movement termination upon contact with an object.

The flat panel detector module was adjusted from the commercial product by the reduction of the back-shielding thickness and the removal of highly attenuating objects (e.g., support screws). This adjusted flat panel detector module has a thickness of $6.8 \mathrm{~cm}$, an average gamma transmission of $52 \%$ at $140 \mathrm{keV}$, and a FOV of $39.9 \times 29.5 \mathrm{~cm}^{2}$. Different from a conventional CBCT scanner was that no anti-scatter grid was present in front of the flat panel detector to increase the gamma photon transmission rate to the gamma camera.

The cone-beam collimator has holes with 40.0-mm length, $1.90-\mathrm{mm}$ inner diameter, and $0.25-\mathrm{mm}$ septal thickness. The gamma camera has a $9.5-\mathrm{mm}$-thick $\mathrm{NaI}(\mathrm{Tl})$ crystal, a 3.9-mm full width at half maximum (FWHM) intrinsic spatial resolution, a 9.4\% energy resolution (at $140 \mathrm{keV}$ ), and a $51.0 \times 38.1$ $\mathrm{cm}^{2}$ effective FOV.

The majority of the $\mathrm{x}$-ray photons is absorbed by the flat panel detector when imaging simultaneously. However, a fraction penetrates the flat panel detector to be absorbed by the gamma camera, possibly deteriorating the gamma camera performance $[10,11]$. In this work, the $\mathrm{x}$-ray tube was translated off-focus from the focal point of the cone-beam collimator by $5 \mathrm{~cm}$ (see Fig. 1b). This off-focus configuration introduces a small distance-dependent mismatch between the fluoroscopic and nuclear projections but greatly reduces the nuclear and fluoroscopic interference. These effects are described in greater detail in the supplementary material.
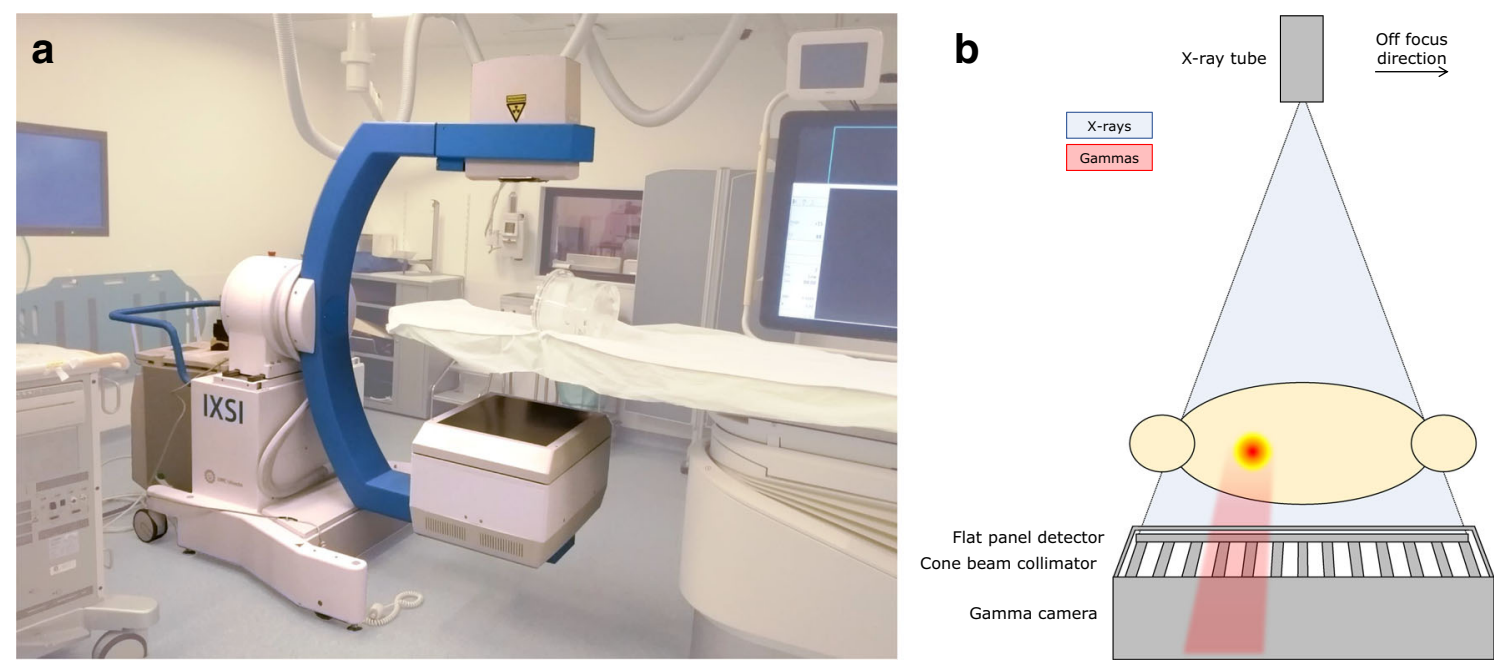

Fig. 1 a IXSI in the intervention room. b A schematic illustration of the IXSI scanner components. For the in-focus configuration, the collimator holes are aligned with the x-ray focal point. For the off-focus configuration, the x-ray tube is shifted by $5 \mathrm{~cm}$ in the indicated direction 


\section{Nuclear image quality}

The nuclear image quality of IXSI was compared to a clinical SPECT/CT scanner (Symbia T16; Siemens Healthineers). The clinical scanner had two detector heads that were mounted with parallel-hole low-energy high-resolution (LEHR) collimators (24.05-mm hole length, 1.11-mm inner diameter, and 0.16-mm septal thickness) and had a $9.5-\mathrm{mm}-$ thick $\mathrm{NaI}(\mathrm{Tl})$ crystal, a 3.9-mm FWHM intrinsic spatial resolution, a $9.9 \%$ energy resolution (at $140 \mathrm{keV}$ ), and a $53.3 \times$ $38.7 \mathrm{~cm}^{2}$ effective FOV.

\section{Planar imaging}

A ${ }^{99 m} \mathrm{Tc}$ point source $(30 \mathrm{MBq}$ ) was positioned at distances from 5 to $40 \mathrm{~cm}$ to the detector (in steps of $5 \mathrm{~cm}$ ) and measured for $1 \mathrm{~min}$. The detector spatial resolution was determined by measuring the FWHM of the Gaussian functions fitted to the projections (for IXSI, divided by the projection magnification from the cone-beam collimator). The detector sensitivity was determined by dividing the total obtained counts by the calibrated source activity.

\section{SPECT}

The NEMA image quality phantom $\left(150 \mathrm{MBq}{ }^{99 \mathrm{~m}} \mathrm{Tc}\right.$ total activity; the spheres filled at an 8:1 concentration ratio) was scanned on both IXSI and the clinical scanner for 10 min over $360^{\circ}$ with 120 angles. Both scanners rotated closely around the phantom. The scans were repeated 5 times (with the acquisition times adjusted to account for radioactive decay) to assess the stability of the reconstructions.

The nuclear projections were, for both scanners, reconstructed with the Utrecht Monte Carlo System iterative reconstruction software package, which includes attenuation compensation, resolution recovery, and Monte Carlo-based scatter correction $[12,13]$. The projections were reconstructed on a $128 \times 128 \times 128$ grid with a 4.79 -mm isotropic voxel size. The reconstructor used the OSEM reconstruction algorithm (6 iterations with 8 subsets) and the reconstructions were smoothed with a 5-mm FWHM Gaussian filter. For the clinical scanner, the attenuation map was retrieved from the clinical software after performing a low-dose CT (made with 110 $\mathrm{kVp}$ at $14 \mathrm{mAs}$ ). For IXSI, the clinical attenuation map was retrieved by registering the $\mathrm{CBCT}$ reconstruction (made with $80 \mathrm{kVp}$ at $73 \mathrm{mAs}$ ) to the attenuation map of the clinical scanner using the elastix software package [14].

The activity recoveries of the spheres in the NEMA image quality phantom (delineated on the low-dose CT reconstruction) were determined as a measure of the reconstruction quality.

\section{Fluoroscopic image quality}

The fluoroscopic image quality of IXSI was compared to a clinical CBCT scanner (Allura Xper FD20; Philips Healthcare). The flat panel detector of the clinical scanner had a FOV of $37.4 \times 28.7 \mathrm{~cm}^{2}$ and was equipped with an anti-scatter grid.

\section{Planar imaging}

An image quality phantom (Fluorad A+D; Pehamad) was imaged with fluoroscopy on both scanners. Both acquisitions were performed using $80 \mathrm{kVp}$ and $2 \mathrm{mAs}$.

\section{CBCT}

An image quality phantom designed for low-contrast liver imaging (Liver Nodule Phantom; QRM) was scanned on both scanners. For IXSI, the acquisition was performed using 80 $\mathrm{kVp}$ and $73 \mathrm{mAs}$ (the same settings that will be used in the planned clinical radioembolization study). For the clinical scanner, the acquisition was performed using $119 \mathrm{kVp}$ and $504 \mathrm{mAs}$ (the same settings that are clinically used for abdomen imaging). The CBCT reconstruction of the clinical scanner was retrieved from the clinical software. The CBCT reconstruction of IXSI was made using the Astra toolbox software package [15] in combination with an image-based scatter correction [16].

\section{Simultaneous planar imaging}

Finally, the potential for simultaneous fluoroscopic and nuclear imaging was demonstrated. An empty syringe of $20 \mathrm{ml}$ was placed in an anthropomorphic thorax phantom (Radiology Support Devices) and during $40 \mathrm{~s}$ filled with a mixture of iodinated contrast and $50 \mathrm{MBq}{ }^{99 \mathrm{~m}} \mathrm{Tc}$ via a connecting tube. The fluoroscopic projections were acquired at $80 \mathrm{kVp}$ ( $27.8 \mathrm{~ms}$ pulse length, $3.75 \mathrm{~Hz}, 1.17 \mathrm{~mA}$ ). The nuclear projections were created with an integration time of $2.7 \mathrm{~s}$ and were smoothed with a 5-mm FWHM Gaussian filter. Digital subtraction images were created by subtracting the fluoroscopic projections obtained during the injection with those acquired before the start of the injection.

\section{Results}

\section{Nuclear image quality}

\section{Planar imaging}

The detector sensitivity and resolution, as a function of the distance of the point source to the detector, are collected in 
Fig. 2a and $\mathrm{b}$ for IXSI and the clinical scanner. IXSI had a lower sensitivity than the clinical scanner due to the increased attenuation from the flat panel detector module, but increased over the source to detector distance due to the converging nature of the cone-beam collimator. IXSI had a worse spatial resolution than the clinical scanner (consistently $\sim 5 \mathrm{~mm}$ larger) due to the extra source to collimator distance arising from the thickness of the flat panel detector module.

\section{SPECT}

Slices of the SPECT reconstructions of the NEMA image quality phantom are shown in Fig. $2 \mathrm{c}$ and d. The clinical scanner acquired $(5.38 \pm 0.02) \times 10^{6}$ nuclear counts, while IXSI acquired $(2.25 \pm 0.01) \times 10^{6}$ counts. The activity recoveries of the NEMA image quality phantom spheres are given in Table 1 . The activity recoveries from IXSI showed $<8 \%$ difference compared with those obtained by the clinical scanner.

\section{Fluoroscopic image quality}

\section{Planar imaging}

The planar images obtained through fluoroscopic imaging are shown in Fig. 3a and b. Visually, the images provided a comparable linearity, contrast, and resolution.

\section{CBCT}

Slices of the CBCT reconstructions of the liver nodule phantom are shown in Fig. 3c and d. IXSI reconstructed a larger field of view than the clinical scanner because IXSI performed a non-circular orbit instead of a circular one. The reconstruction from the clinical scanner provided a better subjective image quality in terms of contrast and uniformity than the IXSI reconstruction because it had fewer scattered photons present in the projections due to the higher $\mathrm{kVp}$ and $\mathrm{mAs}$ setting and the incorporated anti-scatter grid.

\section{Simultaneous planar imaging}

The nuclear, fluoroscopic, and digital subtraction projections obtained during the injection of iodinated contrast with ${ }^{99 \mathrm{~m}} \mathrm{Tc}$ are shown in the supplemental material. Three timeframes are shown in Fig. 4.

\section{Discussion}

This study evaluated the imaging capabilities of a novel hybrid C-arm scanner that has been approved by the local ethics committee to be evaluated in existing clinical workflows. The combination of $\mathrm{x}$-ray and nuclear detection capability in one detector allows for a compact scanner that is compatible with a $\mathrm{C}$-arm which enables a smooth implementation in the intervention room.
Fig. 2 The nuclear image quality evaluation. a The detector sensitivity and (b) the spatial resolution, as a function of the distance from the source to the detector, for IXSI and one detector head of the clinical scanner. c The SPECT reconstruction from IXSI. $\mathbf{d}$ The SPECT reconstruction from the clinical scanner
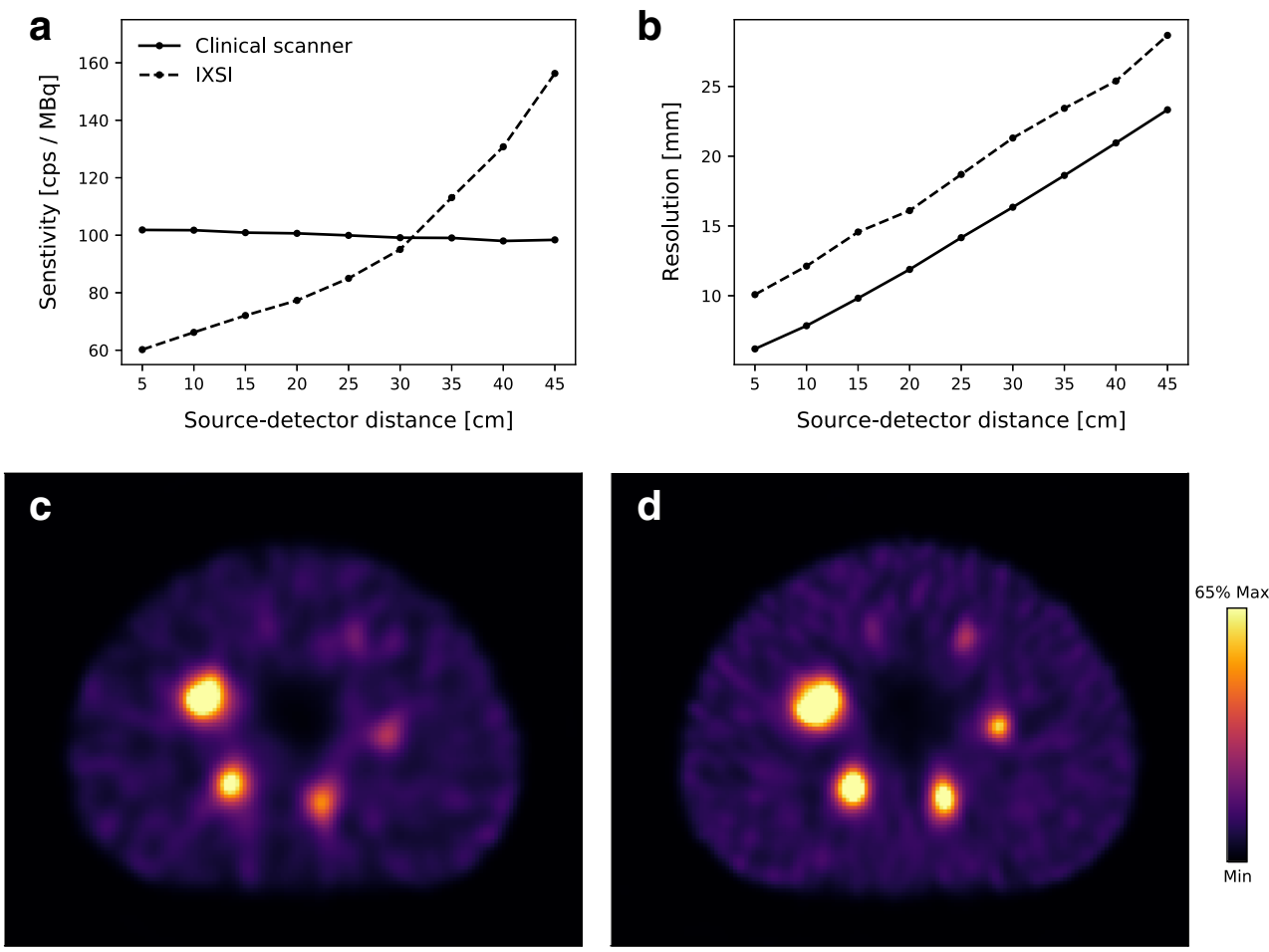
Table 1 The activity recoveries (in percentage) of the NEMA image quality phantom spheres for IXSI and the clinical scanner

\begin{tabular}{lll}
\hline Sphere diameter $(\mathrm{mm})$ & IXSI & Clinical scanner \\
\hline 37 & $72.7 \pm 0.9$ & $74.2 \pm 0.9$ \\
28 & $61.7 \pm 1.1$ & $68.2 \pm 1.0$ \\
22 & $50.4 \pm 4.7$ & $53.3 \pm 2.1$ \\
17 & $37.2 \pm 2.8$ & $44.5 \pm 4.8$ \\
13 & $25.6 \pm 2.9$ & $31.2 \pm 1.7$ \\
10 & $17.9 \pm 2.5$ & $18.8 \pm 2.1$ \\
\hline
\end{tabular}

The phantom experiments showed that the SPECT image quality of IXSI was lower than that of a clinical scanner due to the placement of the flat panel detector in front of the gamma camera and the change from two to one detector head. However, in the case of imaging during radioembolization, it has through digital and experimental studies $[9,17-19]$ been demonstrated that IXSI can be expected to accurately retrieve various clinically and dosimetric important measures. The clinical performance of IXSI in other procedures is under active investigation.
The phantom experiments showed that the CBCT image quality of IXSI was lower than that of a clinical scanner due to scattered photons. For now, the obtained quality is sufficient since we focus on the addition of nuclear images to clinical workflow. In a later stage of the project, we aim to implement the following: (i) a focused anti-scatter grid to reduce the number of scattered photons is reduced, and (ii) a dedicated scatter compensation algorithm in the $\mathrm{CBCT}$ reconstruction.

The scanner will first be employed during the pretreatment procedure of hepatic radioembolization. Simultaneous planar imaging will be used to learn about the potential dynamic uptake behavior of ${ }^{99 \mathrm{~m}} \mathrm{Tc}$-macroaggregated albumin (MAA) particles and a SPECT/CBCT scan will be made to determine dosimetric measures (e.g., the lung-shunting fraction and tumor uptake ratios). With such interventional imaging, it may become possible to merge the pretreatment procedure and the subsequent treatment in a single-session procedure. This innovation could lead to more personalized treatments and will allow for one-stop-shop workflows.

Concluding, a novel hybrid scanner shows promising imaging capabilities for aiding interventions involving radionuclides. Clinical studies are now required for a full evaluation of the device.
Fig. 3 The fluoroscopic image quality evaluation. a The planar fluoroscopic acquisition from IXSI. b The planar fluoroscopic acquisition from the clinical scanner. c The CBCT reconstruction from IXSI. $\mathbf{d}$ The $\mathrm{CBCT}$ reconstruction from the clinical scanner. The white dashed lines indicate the reconstruction field of view
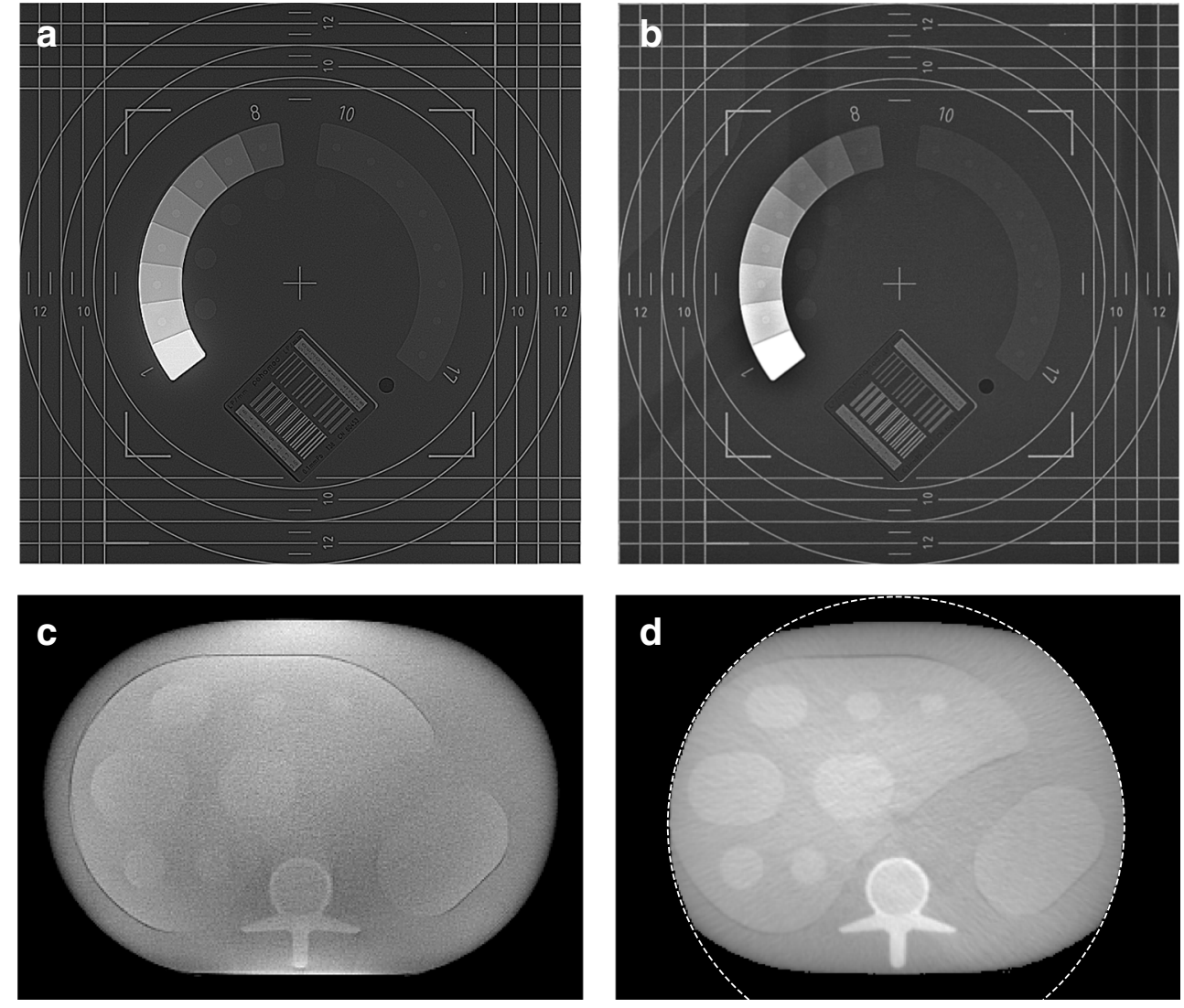
Fig. 4 The projections at three time points $(15,30$, and $40 \mathrm{~s}$ from the start of the injection) that are obtained during the injection of iodinated contrast with ${ }^{99 \mathrm{~m}} \mathrm{Tc}$. Shown are (a) the digital subtraction images, (b) the nuclear projections, and (c) the fluoroscopic projections (in grayscale) merged with the nuclear projections (in color) a
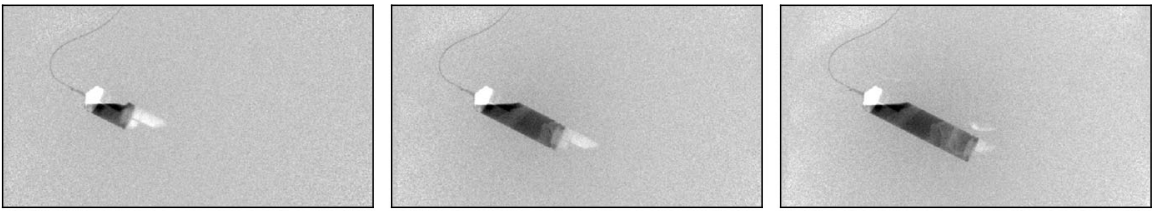

\section{$\prod_{\text {Min }}^{\text {Max }}$}

b
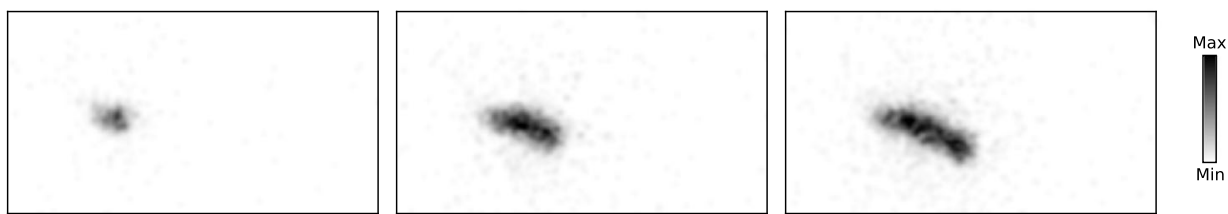

C
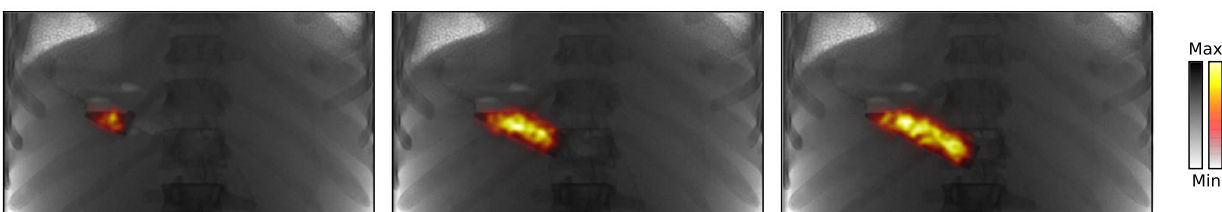

Supplementary Information The online version contains supplementary material available at https://doi.org/10.1007/s00330-021-08023-4.

Funding This study has received funding from the European Union's Horizon 2020 research and innovation program under grant agreements no. 646734 and no. 963934

\section{Declarations}

Guarantor The scientific guarantor of this publication is Hugo WAM de Jong.

Conflict of Interest The authors of this manuscript declare relationships with the following companies: Philips Healthcare.

Statistics and Biometry No complex statistical methods were necessary for this paper.

Informed Consent Written informed consent was not required for this study because this study did not involve human subjects.

\section{Methodology \\ - experimental \\ - performed at one institution}

Open Access This article is licensed under a Creative Commons Attribution 4.0 International License, which permits use, sharing, adaptation, distribution and reproduction in any medium or format, as long as you give appropriate credit to the original author(s) and the source, provide a link to the Creative Commons licence, and indicate if changes were made. The images or other third party material in this article are included in the article's Creative Commons licence, unless indicated otherwise in a credit line to the material. If material is not included in the article's Creative Commons licence and your intended use is not permitted by statutory regulation or exceeds the permitted use, you will need to obtain permission directly from the copyright holder. To view a copy of this licence, visit http://creativecommons.org/licenses/by/4.0/.

\section{References}

1. Vidal-Sicart S, Paredes P, Zanón G et al (2010) Added value of intraoperative real-time imaging in searches for difficult-to-locate sentinel nodes. J Nucl Med 51:1219-1225. https://doi.org/10.2967/ jnumed.110.074880

2. Ortega J, Ferrer-Rebolleda J, Cassinello N, Lledo S (2007) Potential role of a new hand-held miniature gamma camera in performing minimally invasive parathyroidectomy. Eur J Nucl Med Mol Imaging 34:165-169. https://doi.org/10.1007/s00259-006-0239-7

3. Ebbers SC, Barentsz MW, Braat AJAT, Lam MGEH (2019) Intraarterial peptide receptor radionuclide therapy for neuroendocrine tumor liver metastases. Dig Dis Interv 03:81-90. https://doi.org/ 10.1055/s-0038-1676300

4. Braat AJAT, Smits MLJ, Braat MNGJA et al (2015) ${ }^{90} \mathrm{Y}$ hepatic radioembolization: an update on current practice and recent developments. J Nucl Med 56:1079-1087. https://doi.org/10.2967/ jnumed.115.157446

5. Levillain $\mathrm{H}$, Bagni $\mathrm{O}$, Deroose $\mathrm{CM}$ et al (2021) International recommendations for personalised selective internal radiation therapy of primary and metastatic liver diseases with yttrium-90 resin microspheres. Eur J Nucl Med Mol Imaging. https://doi.org/10.1007/ s00259-020-05163-5

6. van der Hoeven BL, Schalij MJ, Delgado V (2012) Multimodality imaging in interventional cardiology. Nat Rev Cardiol 9:333-346. https://doi.org/10.1038/nrcardio.2012.14

7. Beijst C, Elschot M, Viergever MA, de Jong HWAM (2016) Toward simultaneous real-time fluoroscopic and nuclear imaging in the intervention room. Radiology 278:232-238. https://doi.org/ 10.1148/radiol.2015142749

8. van der Velden S, Kunnen B, Koppert WJC et al (2019) A dual-layer detector for simultaneous fluoroscopic and nuclear imaging. Radiology 290:833-838. https://doi.org/10.1148/radiol.2018180796

9. Dietze MMA, Kunnen B, van der Velden S et al (2019) Performance of a dual-layer scanner for hybrid SPECT/CBCT. Phys Med Biol 64: 105020. https://doi.org/10.1088/1361-6560/ab15f6

10. Koppert WJC, van der Velden S, Steenbergen JHL, de Jong HWAM (2018) Impact of intense x-ray pulses on a NaI(Tl)-based 
gamma camera. Phys Med Biol 63:065006. https://doi.org/10. 1088/1361-6560/aaaf02

11. Koppert WJC, Dietze MMA, van der Velden S et al (2019) A comparative study of $\mathrm{NaI}(\mathrm{Tl}), \mathrm{CeBr}$, and $\mathrm{CZT}$ for use in a realtime simultaneous nuclear and fluoroscopic dual-layer detector. Phys Med Biol 64:135012. https://doi.org/10.1088/1361-6560/ ab267c

12. Dietze MMA, van der Velden S, Lam MGEH et al (2018) Fast quantitative reconstruction with focusing collimators for liver SPECT. EJNMMI Phys 5:28. https://doi.org/10.1186/s40658-0180228-5

13. Dietze MMA, Kunnen B, Stella M, de Jong HWAM (2020) Monte Carlo-based scatter correction for the SMARTZOOM collimator. EJNMMI Phys 7:49. https://doi.org/10.1186/s40658-020-00318-7

14. Klein S, Staring M, Murphy K et al (2010) elastix: a toolbox for intensity-based medical image registration. IEEE Trans Med Imaging 29:196-205. https://doi.org/10.1109/TMI.2009.2035616

15. van Aarle W, Palenstijn WJ, Cant J et al (2016) Fast and flexible Xray tomography using the ASTRA toolbox. Opt Express 24:25129 25147. https://doi.org/10.1364/OE.24.025129
16. Rührnschopf E-P, Klingenbeck K (2011) A general framework and review of scatter correction methods in X-ray cone-beam computerized tomography. Part 1: Scatter compensation approaches. Med Phys 38:4296-4311. https://doi.org/10.1118/1.3599033

17. Dietze MMA, Bastiaannet R, Kunnen B et al (2019) Respiratory motion compensation in interventional liver SPECT using simultaneous fluoroscopic and nuclear imaging. Med Phys 46:3496-3507. https://doi.org/10.1002/mp.13653

18. van der Velden S, Dietze MMA, Viergever MA, de Jong HWAM (2019) Fast technetium-99m liver SPECT for evaluation of the pretreatment procedure for radioembolization dosimetry. Med Phys 46:345-355. https://doi.org/10.1002/mp.13253

19. Dietze MMA, Kunnen B, Lam MGEH, de Jong HWAM (2021) Interventional respiratory motion compensation by simultaneous fluoroscopic and nuclear imaging: a phantom study. Phys Med Biol 66:065001. https://doi.org/10.1088/1361-6560/abe556

Publisher's note Springer Nature remains neutral with regard to jurisdictional claims in published maps and institutional affiliations. 